\title{
Porous polydopamine and para-aminobenzoic acid complex membrane formed by electrochemical codeposition/degradation
}

\author{
Hongfei Shao ${ }^{1, a}$, Huaixiang $\mathrm{Li}^{2, b}$ and Wei $\mathrm{He}^{3, \mathrm{c}}$ \\ ${ }^{1}$ Shandong Non-metal material institute, Jinan, China \\ ${ }^{2}$ Shandong Normal University, Jinan, China \\ ${ }^{3}$ Shandong University, Jinan, China

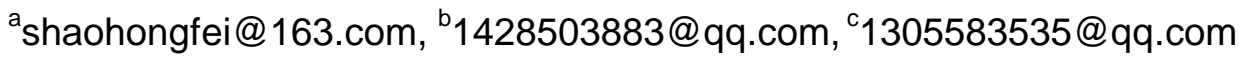

\begin{abstract}
Keywords: electropolymerization, electrochemical degradation, porou polydopamine, dopamine determination

Abstract. Electropolymerization is a useful method to modify electrode for improving selectivity, sensitivity and strong adherence to electrode surface. In this paper, we prepared polydopamine (PDA), poly-para-aminobenzoic acid (PABA) and PDA/PABA composite membrane on a glassy carbon electrode (GCE) using cyclic voltammetry. The composite membrane was electrochemically degraded to form a porous polydopamine membrane (PPM) on the GCE. The porosity of the PPM was examined using anionic redox couple of ferricyanide/ferrocyanide. PPM modified electrodes were able to give better voltammetric responses for dopamine (DA) oxidation than the single PDA and PABA modified ones.
\end{abstract}

\section{Introduction}

It is reported that para-aminobenzoic acid (p-ABA) could be easily electropolymerized on various substrate materials to form poly-para-aminobenzoic acid (PABA) films with good chemical and mechanical stability [1-3]. Since the amino group is an important functional group in PABA that undergoes oxidation on the electrode surface, the modification of the electrode surface is supposed to increase the sensitivity of it [4,5]. However, electrochemical synthesis of PDA/PABA blend membranes followed by electrochemical degradation to produce porous polydopamine membranes (PPM) has not been reported yet as far as we know. This basic information is useful and important for us to explore the fundamental characteristics of PDA/polymer blend membranes and to further optimize their performances for diverse potential applications in water treatment, bioseparation and blood purification [6].

Dopamine (DA) is an important catecholamine neurotransmitter compound that has been paid much attention recently. It plays a significant role in the function of the central nervous, renal, hormonal and cardiovascular system [7]. Trace level measurement of DA has been a long-standing goal since the concentration change is associated with various diseases and disorders, such as Parkinson's disease, Alzheimer's disease, Huntington's disease, epilepsy, pheochromocytoma and neuro- blastoma [8]. It is important to monitor DA sensitively and selectively not only for biomedical chemistry and neurochemistry research but also for diagnostic and pathological purpose [9]. Different methods have been used for the determination of DA such as spectrophotometry [10], fluorescence method [11,12], electrochemiluminescence [13,14], high performance liquid chromatography (HPLC) [15,16], capillary electrophoresis [17], molecular imprinting polymer [18-20], complementary metal oxide semiconductor (CMOS) capacitive sensors [21,22], photoelectrochemical sensors [23,24] and electrochemical method [25-27]. Among the modified electrodes, polymer modified electrodes (PMEs) prepared by electropolymerization have been drawn extensive attention due to their high selectivity, sensitivity and strong adherence to electrode surface $[28,29]$. 


\section{Experimental}

Materials and apparatus. Dopamine (DA) hydrochloride, para-aminobenzoic acid (p-ABA), potassium ferricyanide, $\mathrm{Na}_{2} \mathrm{HPO}_{4} \cdot 12 \mathrm{H}_{2} \mathrm{O}, \mathrm{NaH}_{2} \mathrm{PO}_{4} \cdot 2 \mathrm{H}_{2} \mathrm{O}$, and other reagents were obtained from Sinopharm Chemical Reagent Co. Ltd. (Shanghai, China). All other reagents were of at lease analytical-reagent grade and were dissolved in doubly distilled water (DDW). A phosphate buffer solution (PBS) was made of $0.1 \mathrm{M} \mathrm{Na}_{2} \mathrm{HPO}_{4}$ and $0.1 \mathrm{M} \mathrm{NaH}_{2} \mathrm{PO}_{4}$ and adjusted to $\mathrm{pH}=7$ either using $\mathrm{NaOH}(0.1 \mathrm{M})$ or $\mathrm{HCl}(0.1 \mathrm{M})$. All cyclic voltametry $(\mathrm{CV})$ experiments were performed on a LK2005 electrochemical workstation (Lanlike Company, Tianjin, China). A conventional three-electrode system was applied. The working, the auxiliary, and the reference electrodes were modified glassy carbon electrode (GCE, diameter: $3 \mathrm{~mm}$ ), platinum plate, and saturated calomel electrode (SCE), respectively.

Electropolymerization of membranes. Prior to experiment, a GCE with the diameter of $3.0 \mathrm{~mm}$ was polished with diamond paste and ultrasonic cleaned sequentially in acetone, $\mathrm{NaOH}(1.0 \mathrm{M})$, $\mathrm{HNO}_{3}(1: 1, \mathrm{v} / \mathrm{v})$ and doubly distilled water. polydopamine (PDA), poly-para-aminobenzoic acid (PABA) PABA, and PDA-PABA composite membranes were prepared by cycling the electrode potential between -0.60 and $+1.00 \mathrm{~V}$ (vs. SCE), $25 \mathrm{CV}$ scans at the scan rate of $50 \mathrm{mV} \cdot \mathrm{s}^{-1}$ in $0.1 \mathrm{M}$ PBS containing $6 \mathrm{mM}$ DA, $6 \mathrm{mM}$ p-ABA, and $3 \mathrm{mM}$ DA + $3 \mathrm{mM}$ p-ABA, respectively.

Membrane degradation by $\mathrm{CV}$ in $\mathrm{KOH}$ solution. The electrosynthesized PDA, PABA and PDA+PABA films were electrochemically degraded by cycling the potential between -0.60 and 1.20 $\mathrm{V}$ (vs. SCE), $2 \mathrm{CV}$ scans in $0.05 \mathrm{M} \mathrm{KOH}$ solution at the scan rate of $80 \mathrm{mV} \cdot \mathrm{s}^{-1}$ to produce porous polydopamine membranes (PPM) on the GCE.

Characterization of the membrane. Electrochemical characterizations were also made by the CV technique. A CV cycle was realized between -0.80 and $0.80 \mathrm{~V}$ (vs. SCE), at a scan rate of 20 $\mathrm{mV} \cdot \mathrm{s}^{-1}$. For these experiments, the electrolyte is a solution of potassium hexacyanoferrate trihydrate at $3 \mathrm{mM}$ (Sigma Aldrich) containing potassium chloride $(0.10 \mathrm{M})$. A glassy carbon plate electrode of surface area $1.0 \mathrm{~cm}^{2}$ was utilized for elecrodeposition/electrodegradation and used for X-ray photoelectron spectroscopy (XPS) measurements samples. XPS measurements were performed on a Perkin Elmer PHI-5600 system (USA) with monochromatic Al K $\alpha(\mathrm{h} v=1486.71 \mathrm{eV}) \mathrm{X}$-ray radiation $(15 \mathrm{kV}$ and $10 \mathrm{~mA})$ and hemispherical electron energy analyzer. All XPS spectra were corrected according to the $\mathrm{C} 1 \mathrm{~s}$ line at $284.6 \mathrm{eV}$.

Electrochemical measurements for selective detection DA. Standard solution of DA and AA were prepared using DDW as solvent and stored under $4{ }^{\circ} \mathrm{C}$. Electrochemical analysis of DA on the modified electrodes was carried out in $0.10 \mathrm{M}$ PBS containing a certain amount of DA. The CV cycles were realized between -0.6 and $0.6 \mathrm{~V}$ (vs. SCE), at a scan rate of $50 \mathrm{mV} \cdot \mathrm{s}^{-1}$. After each measurement, the modified electrode was polished and washed with water and scanned in blank PBS until the electrochemical signals of DA were disappeared.

\section{Results and discussion}

Electropolymerization of membranes. Fig. 1 shows the cyclic voltammograms of electrosynthesis of PDA-PABA films in $0.1 \mathrm{M}$ PBS containing $\mathrm{mM} \mathrm{DA}+3 \mathrm{mM}$ PABA at a scan rate of $50 \mathrm{mV} \cdot \mathrm{s}^{-1}$, 25 scan cycles. The heights of all current peaks decreased cycle-by-cycle, which suggested the formation of a compact and insulating film which progressively covered the electrode surface, leading to the suppression of the voltammetric response. 


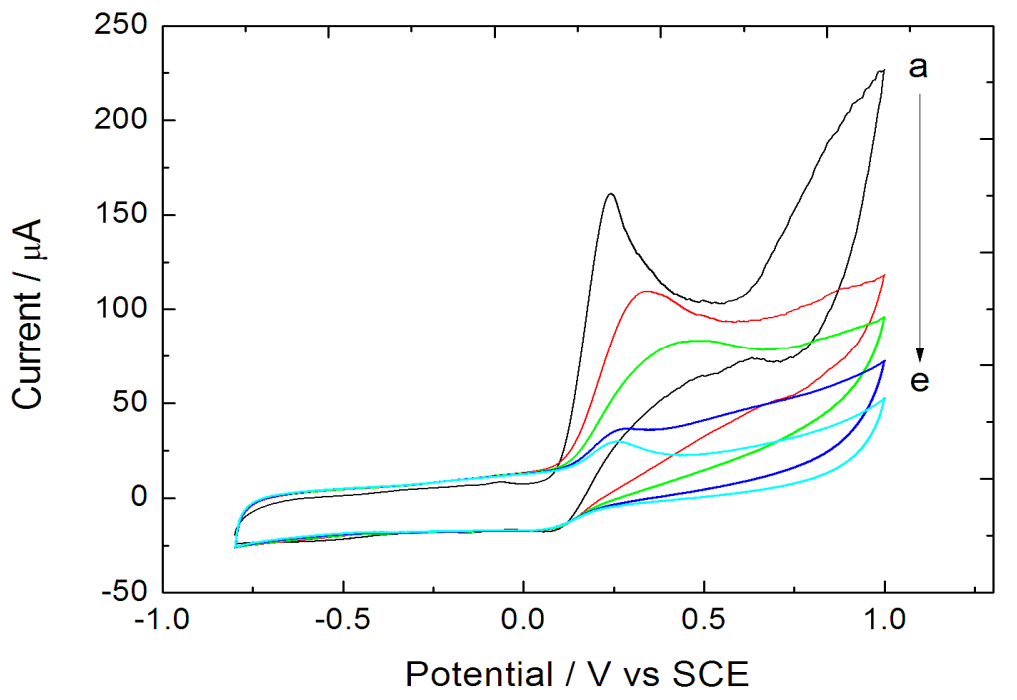

Fig. 1. Cyclic voltammograms for the electropolymerization of $3 \mathrm{mM}$ dopamine + $3 \mathrm{mM}$ p-ABA (C) in a $0.1 \mathrm{M}$ PBS (pH 7.0) on a GCE. Scan rate: $50 \mathrm{mV} \cdot \mathrm{s}^{-1}$; number of scan cycles: 25. Number of cycles: a 1, b 4, c 7, d 12, e 25.

Characterization of electrochemical degraded of membranes. Membranes of PDA+PABA deposited by electrochemistry are degraded by cyclic voltammetry in a solution of potassium hydroxide. The degradation was performed by two cycling the potential between -0.60 and $1.20 \mathrm{~V}$ (vs . $\mathrm{SCE}$ ) in $0.05 \mathrm{M} \mathrm{KOH}$ solution. After degradation, a characterization by voltammetry is made in the presence of a potassium hexacyanoferrate trihydrate $(3 \mathrm{mM})$ solution containing potassium chloride $(0.10 \mathrm{M})$.

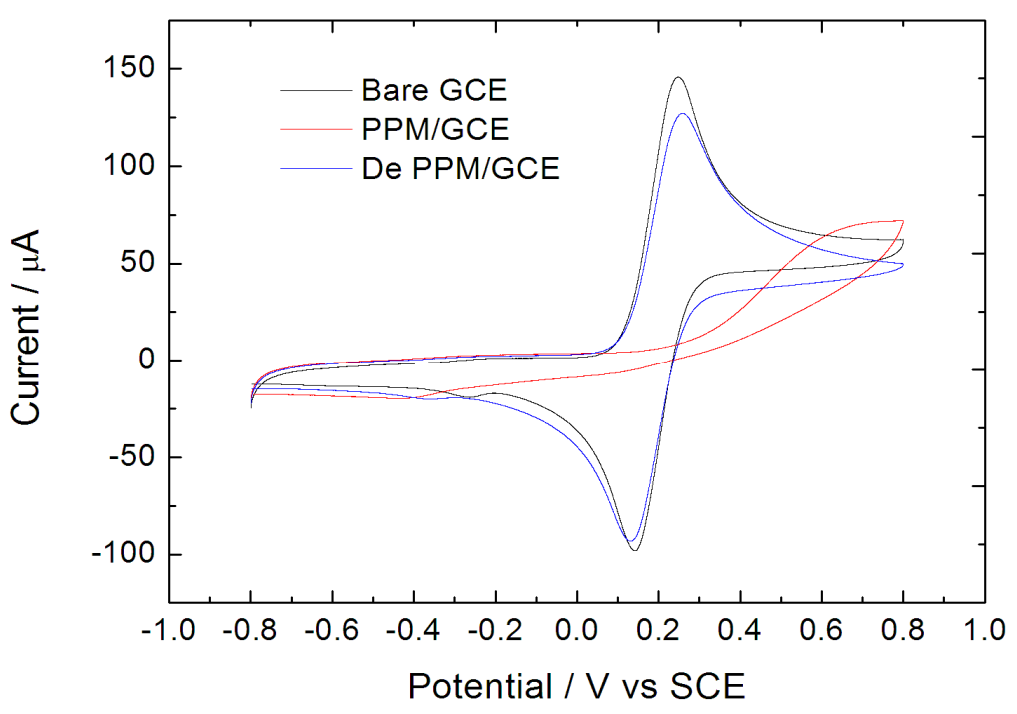

Fig. 2. Cyclic voltammograms of $5 \mathrm{mM} \mathrm{K} \mathrm{K}_{3} \mathrm{Fe}(\mathrm{CN})_{6} / \mathrm{K}_{4} \mathrm{Fe}(\mathrm{CN})_{6}$ in deoxygenated $0.10 \mathrm{M} \mathrm{KCl}$ supporting electrolyte, recorded at bare GCE (black line ), PDA+PABA/GCE (red line),PPM/GCE (blue line).

Fig. 2 presents the characterizations of cyclic voltammograms with the $\mathrm{Fe}(\mathrm{CN})_{6}{ }^{3-} / \mathrm{Fe}(\mathrm{CN})_{6}{ }^{4-}$ redox activity at a bare GCE as a reference object. A couple of typical redox peaks of hexacyanoferrate appears on the bare GCE (black curve). The the couple of redox peaks of hexacyanoferrate at degraded PABA/GCE is almost recover that at the bare GCE, which reveals that the PABA membrane 
was almost fully degraded by electrochemical cycling in the $\mathrm{KOH}$ solution. As we can see from Fig. 2, a compact layer at the surface of the working electrode impeding the access of the redox couple of ferricyanide/ferrocyanide to the electrode (red curve). However, an obvious current increase appears when using the degraded PPM/GCE under same experimental conditions. Although the modified layer insulates the GCE to a large extent, the redox peak current can still be seen in the voltammogram ((blue curve). This may result from the cavities caused by the electrochemical degradation of PABA to form some channels for hexacyanoferrate to arrive at the electrode surface. Certainly, the peak currents clearly are smaller than those at the bare GCE. These results are accordant with XPS observation as described in Fig. 3 as follows.
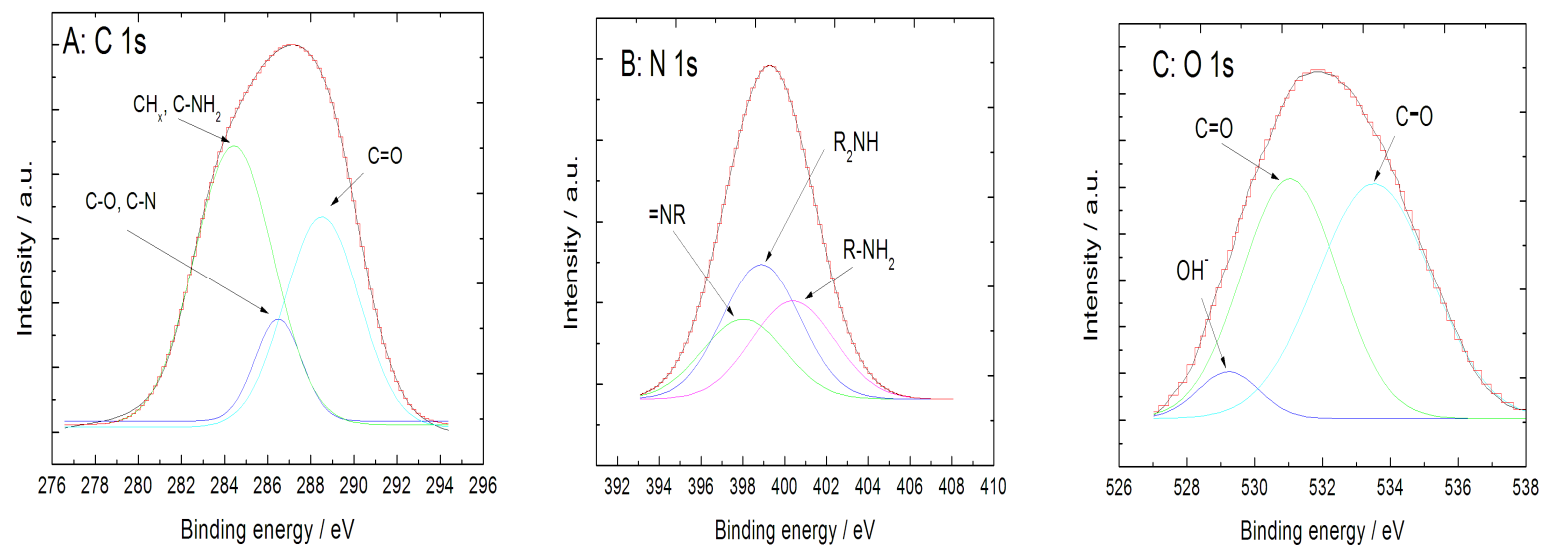

Fig. 3. High-resolution XPS spectra of C 1s, N 1s, and O 1s regions for a porous polydopamine membrane.

Fig. 3 shows XPS measurements provided chemical state information of the PPM and representative high-resolution spectra of the $\mathrm{C} 1 \mathrm{~s}, \mathrm{~N} 1 \mathrm{~s}$, and $\mathrm{O} 1 \mathrm{~s}$ regions. Deconvolution of the $\mathrm{C} 1 \mathrm{~s}$ signal in Fig. 3(A) shows three peaks assigned to $\mathrm{CHx} / \mathrm{C}-\mathrm{NH} 2, \mathrm{C}-\mathrm{O} / \mathrm{C}-\mathrm{N}$, and $\mathrm{C}=\mathrm{O}$ species [30]. Fig.3(B) shows the three components of $\mathrm{N}$ 1s peak corresponding to imino groups (=N-R, $398.5 \mathrm{eV})$, substituted amines (R-NH-R or indole groups, $399.8 \mathrm{eV})$, and amine groups (R-NH2, $401.7 \mathrm{eV})$ [31]. The peak positions and relative amount of $\mathrm{N}$ 1s components in PPM resembles those of the synthetic PDA film on silica wafer [32]. In Fig. 3(C), the O 1s spectrum of PPM is deconvoluted into three components of $\mathrm{OH}-$ at $529.2 \mathrm{eV}, \mathrm{C}=\mathrm{O}$ at $531.1 \mathrm{eV}$, and $\mathrm{C}-\mathrm{O}$ at $533.3 \mathrm{eV}$, which are consistent with those of C 1s [33].

Electrochemical behavior of DA and AA at the modified electrodes. The PPM/GCE was then taken as a possible application for DA sensing in $\mathrm{pH}$ 7.0 PBS. The cyclic voltammogram of $10 \mu \mathrm{M}$ DA (Fig.4 A) exhibited a pair of redox peaks, attributed to the electron-transfer reaction of dopamine/dopaminechrome [3]. At bare GCE (curve a), very small peak currents were obtained. The PDA/GCE (curve b) and PABA/GCE (curve c) showed some electrocatalysis of DA by increasing oxidation currents. The proposed PPM/GCE (curve d) showed the largest peak currents in comparison with the other electrodes. The improved oxidation reaction of DA at PPM/GCE was interpreted due to the possible recognition mechanisms. First, the adsorption in the effective micro-cavities structure. Secondly, the randomly distribution and high density of oxygen groups of the PPM enable the interaction with $-\mathrm{NH}_{2}$ and $-\mathrm{OH}$ groups in DA through electrostatic interaction and/or hydrogen bonding [34].

The electrochemical performance of an important anionic interference, $100 \mu \mathrm{M}$ AA, using the proposed electrodes were further evaluated. As shown in Fig.4 (B), at bare GCE (curve a), a moderate oxidation peak current at about $-0.05 \mathrm{~V}$ was observed. The PDA/GCE (curve b) showed small oxidation currents at about $0.10 \mathrm{~V}$. The highest oxidation current was observed at PABA/GCE (curve c). This indicates that the single modification by PDA or PABA could not eliminate interference of AA. As a comparison, the electrochemical activity of AA is very weak at the PPM/GCE (curve d). 
The results demonstrate that co-deposition of PDA/PABA membrane followed by electrochemically degradation is effective in lowering the interference from AA.
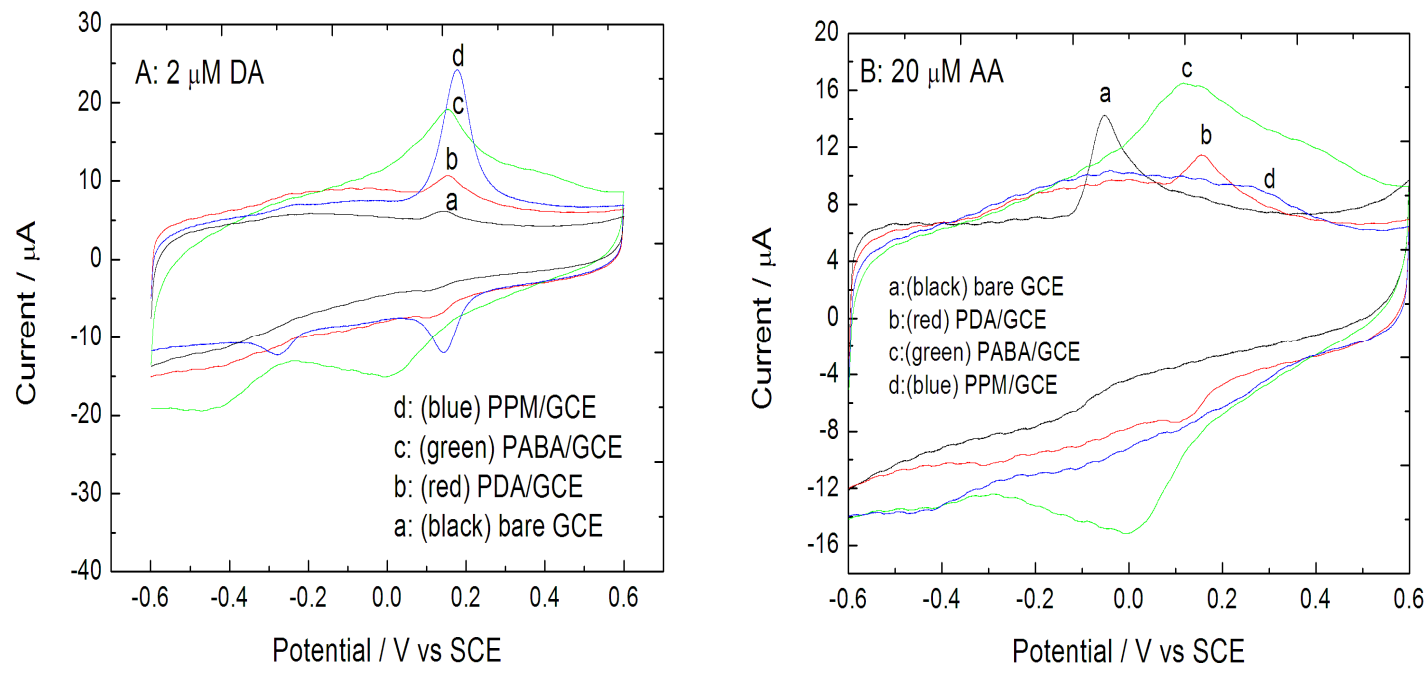

Fig. 4. (A) CVs of $10 \mu \mathrm{M}$ DA in pH 7.0 PBS based on bare GCE (a: black)), PDA/GCE (b: red), PABA/GCE (c: green) and PPM/GCE (d:blue); (B) CVs of $20 \mu \mathrm{M}$ AA based on bare GCE (a: black), PDA/GCE (b: red), PABA/GCE (c: green) and PPM/GCE (d: blue) Scan rate: 50 $\mathrm{mV} \cdot \mathrm{s}^{-1}$.

Stability and reusability of the PPM/GCE. For a DA level of $10 \mu \mathrm{M}$, the relative standard deviation of 20 successive measurements $C V$ response was 3.3\%. When not used, the PPM/GCE was stored in $0.1 \mathrm{M}$ PBS (pH 7). No obvious change in the anodic peak current of DA was observed after two weeks at room temperature, indicating that the PPM was very stable.

\section{Conclusions}

A new type of electrochemical sensor for determination of DA based on porous polydopamine membrane modified glassy carbon electrode has been proposed in this work. The porous polydopamine membrane was formed by electrically polymerizing PDA and PABA composite and then gradually electrochemically degrading PABA. Results show that porous polydopamine membrane can inhibit both AA and UA electrochemical activity. Moreover, the structure of the porous polydopamine membrane maintains a long stability for measurements and storage.

\section{Acknowledgements}

This work was financially supported by the National Natural Science Foundation of China (No: 21405096; 21175084).

\section{Reference}

[1] X.L. Xu, F. Huang, G.L. Zhou, S. Zhang and J.L. Kong: Sensors Vol.10 (2010) , p. 8398.

[2] Y.T. Liu, J. Deng, X.L. Xiao, L. Ding, Y.L. Yuan, H. Li, X.T. Li, X.N. Yan and L.L. Wang: Electrochim. Acta Vol.56 (2011), p. 4595.

[3] F. Xu, M. Gao, L.Wang, G. Shi, W. Zhang, L. Jin and J. Jin: Talanta Vol.55 (2001) , p. 329.

[4] R.M. Kotkar, A.K. Srivastava: Sens. Actuators B Vol.119 (2006), p. 524. 
[5] L. Shang, F. Zhao and B. Zeng: ACS Appl. Mater. Interfaces Vol.6 (2014), p. 18721.

[6] J.H. Jiang, L.P. Zhu, H.T. Zhang, B.K. Zhu andY.Y. Xu: J. Membr. Sci Vol.457 (2014) , p. 73.

[7] Y. Wang, Y. Li, L. Tang, J. Lu and J. Li: Electrochem. Commun Vol.11 (2009), p. 889.

[8] L. Liu, J. Du, S. Li, B. Yuan, H. Han, M. Jing and N. Xia: Biosens. Bioelectron Vol.41 (2013), p. 730.

[9] C. Wang, J. Du, H. Wang, C. Zou, F. Jiang, P. Yang and Y. Du: Sens. Actuators B Vol.204 (2014), p. 302.

[10] J.F. van Staden, R.I.S. van Staden: Talanta Vol.102 (2012) , p. 34.

[11] Y. Zhao, S. Zhao, J. Huang and F. Ye: Talanta Vol.85 (2011) , p. 2650.

[12] B. Aswathy, G. Sony: Microchem. J Vol.116 (2014) , p. 151.

[13] B. Wu, C. Miao, L. Yu, Z. Wang, C. Huang and N. Jia: Sens. Actuators B Vol.195 (2014) , p. 22.

[14] D. Yuan, S. Chen, R. Yuan, J. Zhang and X. Liu: Sens. Actuators B Vol.191 (2014) , p. 415.

[15] H.R. Kim, T.H. Kim, S.H. Hong and H.G. Kim: Biochem. Biophys. Res. Commun Vol.419 (2012), p. 632.

[16] G.E. De Benedetto, D. Ficoa, A. Pennetta, C. Malitesta, G. Nicolardi, D.D Lofrumento, F. De Nuccio and V. La Pesa: J. Pharm. Biomed. Anal Vol.98 (2014), p. 266.

[17] Y. Wang, H. Chen: Journal of Chromatogr. A Vol.1080 (2005) , p. 192.

[18] T.S. Anirudhan, S. Alexander and A. Lilly: Polymer Vol.55 (2014) , p. 4820.

[19] J. Li, J. Zhao and X. Wei: Sens. Actuators B Vol.140 (2009) , p. 663.

[20] T. Qian, C. Yu, X. Zhou, P. Ma, S. Wu, L. Xu and J. Shen: Biosen. Bioelectron Vol.58 (2014), p. 237.

[21] M.S.C. Lu, Y.C. Chen and P.C. Huang: Biosen. Bioelectron Vol.26 (2010) , p. 1093.

[22] S. Casalini, F. Leonardi, T. Cramer and F. Biscarini: Org. Electron Vol.14 (2013) , p. 156.

[23] G.L. Wang, H.J. Jiao, K.L. Liu, X.M. Wu, Y.M. Dong, Z.J. Li and C. Zhang: Electrochem. Commun Vol.41 (2014), p. 47.

[24] Y. Yan, Q. Liu, X. Du, J. Qian, H. Mao and K. Wang: Anal. Chim. Acta Vol.853 (2015), p. 258.

[25] T. Yin, W. Wei and J. Zeng: Anal. Bioanal. Chem Vol.386 (2006) , p. 2087.

[26] S.S. Shankar, B.E. Kumara Swamy and B.N. Chandrashekar: J. Mol. Liq Vol.168 (2012), p. 80.

[27] M. Ahn, J. Kim: J. Electroanal. Chem Vol.683 (2012) , p. 75.

[28] W. Kang, H. Li, M. Ai, S. Wei, H. Gao, J. Liu and Y. Qian: Mater. Lett Vol.116 (2014), p. 374.

[29] T. Qian, C. Yu, X. Zhou, S. Wu and J. Shen: Sens. Actuators B Vol.193 (2014), p. 759.

[30] R.A. Zangmeister, T.A. Morris and M.J. Tarlov: Langmuir Vol.29 (2013), p. 8619.

[31] F. Bernsmann, A. Ponche, C. Ringwald, J. Hemmerlé, J. Raya, B. Bechinger, J.C. Voegel, P. Schaaf and V. Ball: J. Phys. Chem. C Vol.113 (2009), p. 8234.

[32] H. Gao, Y. Sun, J. Zhou, R. Xu and H. Duan: ACS Appl. Mater. Interfaces Vol.5 (2013) , p. 425.

[33] J.M. Lin, Y.L. Su, W.T. Chang, W.Y. Su and S.H. Cheng: Electrochim. Acta Vol.149 (2014), p. 65.

[34] J. Li, X. Lin: Sens. Actuators B Vol.124 (2007) , p. 486. 